\title{
Caries inhibition around Gallium alloy by fluoride releasing resin cement
}

\author{
Nasman Nur alim \\ Department of Oral Surgery Faculty of Dentistry Universitas Hasanuddin
}

\section{ABSTRACT}

Fluoride releasing materials inhibit secondary caries. Gallium alloy has been developed to replace mercury based amalgam. The purpose of this study was to test a new $\mathrm{F}$ releasing resin-ionomer cement for inhibition of 24 extracted human premolars. The experimental cavity (ARG) were filled using etching, priming, and $\mathrm{F}$ releasing resin-ionomer cement (All-bond 2 \& Presinomer, Bisco) followed by condensation of gallium alloy (G Tokuriki Honten, Japan). Three different controls were used: gallium alloy only (G), no etching, Presinomer, gallium alloy (RG), etching, priming, non-F cement (All-bond C\&B, Bisco) and gallium alloy (ACG) The teeth were thermocycled 500x, stored in humidor 28 days, then exposed to artificial caries for 21 days using a strep. mutans culture. Next they were sectioned and examined by microradiography. The microradiographs were examined for presence of a caries inhibition zone near the restoration and classified as strongly inhibited $(\mathrm{SI})$, moderately inhibited $(\mathrm{MI})$ or not inhibited $(\mathrm{N})$ at the enamel and dentin wall. A Chi-square analysis showed that $G$ is different from ARG, ACG is different from ARG, and RG is different from ACG $(p<0.05)$. The results show that the fluoride releasing resin-ionomer cement provided caries inhibition with or without etching and bonding, and that etching and bonding alone is not as effective as fluoride release.

Key words: Secondary caries in vitro, gallium alloy, fluoride releasing resin-ionomer cement

\section{INTRODUCTION}

Secondary caries is one of the main factors for the replacement of amalgam restorations. Amalgam restorations were previously dependent upon mechanical retention, but can be done adhesively now. It is frequently observed that the margin of amalgam filling is partially fractured and the width of the gap between amalgam and tooth gradually increases with time. Many investigators have reported that oral microorganisms penetrate into the ditched margins and the gap, resulting in secondary caries with outer lesions and wall lesions. $^{1-3}$

Recently, improvements of bonding systems have resulted in increased bond strength between amalgam and tooth, and improved resistance to microleakage. Previous studies showed that the adhesive amalgam technique used with a resin cement had the effect of inhibiting secondary caries. ${ }^{4}$

It is known that fluoride releasing materials inhibit secondary caries. Resin cement containing a glass ionomer filler has a good adhesive bonding and a highly effective seal against microleakage for the adhesive amalgam technique. ${ }^{5}$ 
However, it is not yet clear whether such a resin ionomer provides protection against secondary caries.

Dental amalgam contains mercury, which has raised concerns about possible its effects on health. Gallium alloy has been developed to replace mercury based amalgam. In this study, we used gallium alloy as a restorative material.

The purpose of this study was to test a new fluoride releasing resin-ionomer cement for inhibition of secondary caries around gallium alloy fillings. We evaluated the presence of caries inhibition zone adjacent to restorative materials by microradiography and investigated the distribution of calcium; phosphate and fluoride at the demineralized or intact areas of tooth substrates around restorative materials by electron probe microanalysis.

\section{MATERIALS AND METHODS}

Twenty four freshly extracted first upper permanent premolars, free of caries and other defects, were selected for in vitro secondary caries procedures. Sample teeth were divided into three groups randomly of eight each. The teeth were carefully cleaned, the roots were cut off and the pulp tissue was removed. Two class $V$ cavities were prepared at the cementoenamel junction on facial and lingual surfaces of each tooth using a high-speed diamond point with water coolant. The depth of each cavity was standardized at 1.5$2 \mathrm{~mm}$ and the width occlusogingivally $2 \mathrm{~mm}$.

Table 1 shows the materials used in this study. The experimental cavity (ARG) were filled using etching, priming, and $F$ releasing resin-ionomer cement (All-bond 2 \& Presinomer) followed by condensation of gallium alloy (Gallium alloy GF II). Three different controls were used: gallium alloy only $(G)$, no etching, Presinomer, gallium alloy (RG), etching, priming, non-F cement (Allbond $(\& B)$ and gallium alloy (ACG) as shown in Fig. 1 All restorative procedures were applied in accordance with the manufacturer's instructions.

The restorations were polished with a white carborundum point under water spray after storage at $37^{\circ} \mathrm{C}, 100 \%$ humidity for 24 hours. The teeth were thermocycled 500x and were stored in the humidor 28 days.

\section{In vitro secondary caries procedures}

The tooth surfaces were coated with a nail varnish except the peripheral area of about 0.5 $\mathrm{mm}$ around the margins of the restorations. The specimens were mounted on plastic tubes and sterilized with an ethylene-oxide gas. They were inserted into an artificial caries chamber ${ }^{4,6}$ and suspended in sterilized brain-hart infusion broth (Difco Laboratories, Detroit, MI, USA) containing $0.5 \%$ yeast extract and $1 \%$ sucrose. Hanks' BSS (Nacalai tesque, Kyoto, Japan) was poured into each tube. The broth was inoculated with Streptococcus mutans IFO 13955 and incubated at $37^{\circ} \mathrm{C}$. The medium in the chamber was replaced for every 3-4 days and Hanks' BSS in the tube was changed every 7 days. After incubation for 21 days, the specimens were removed and embedded in an epoxy resin.

\section{Examination of caries inhibition zones by microradiography}

The teeth were cut longitudinally through both restorations, and ground sections about 60-80 $\mu \mathrm{m}$ in thickness were prepared from each specimen. A contact microradiograph was taken with soft x-ray (Softex CMR-2, Softex Co, Tokyo, Japan) at $16 \mathrm{kVp}, 3 \mathrm{~mA}$ and were examined with a light microscope at $100 x$. The microradiographs were examined for presence of caries inhibition zones near the restoration shown in Fig. 2 and classified

Table 1. Materials used in this study.

\begin{tabular}{ccc}
\hline Material & Manufacturer & Lot.No \\
\hline All-bond 2 & Bisco, USA & \\
All-Etch & & 059024 \\
Primer A & & 059024 \\
Primer B & & 059104 \\
& & \\
All-bond C \& B & Bisco, USA & \\
LUTING CEMENT & & 089244 \\
Base & & 089234 \\
Catalyst & & \\
& & 019165 \\
Presinomer & Bisco, USA & 109314 \\
Base & & \\
Catalyst & & 9630011107 \\
\hline Gallium alloy & Tokuriki Honten, Japan & \\
GF II & &
\end{tabular}




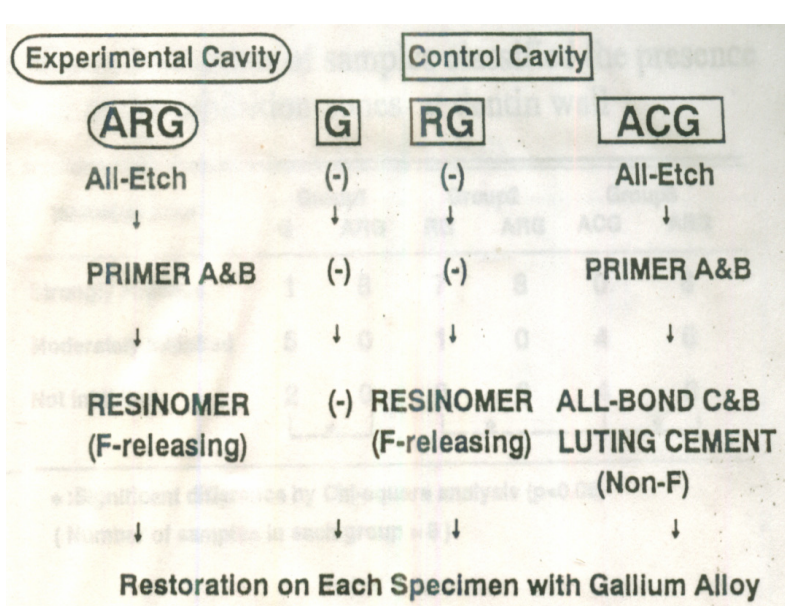

Figure 1. Procedure for the treatment of cavity surfaces.

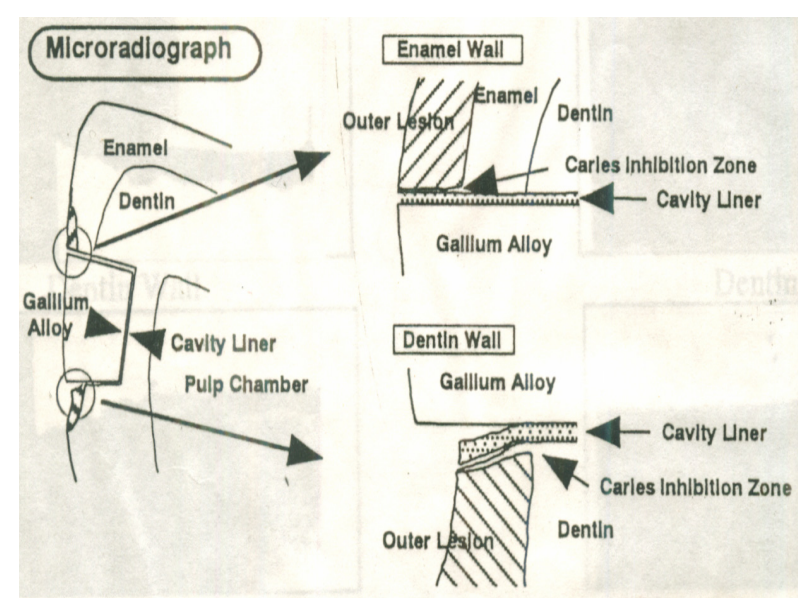

Figure 2. Examination for the presence of caries inhibition zones by microradiopraph.

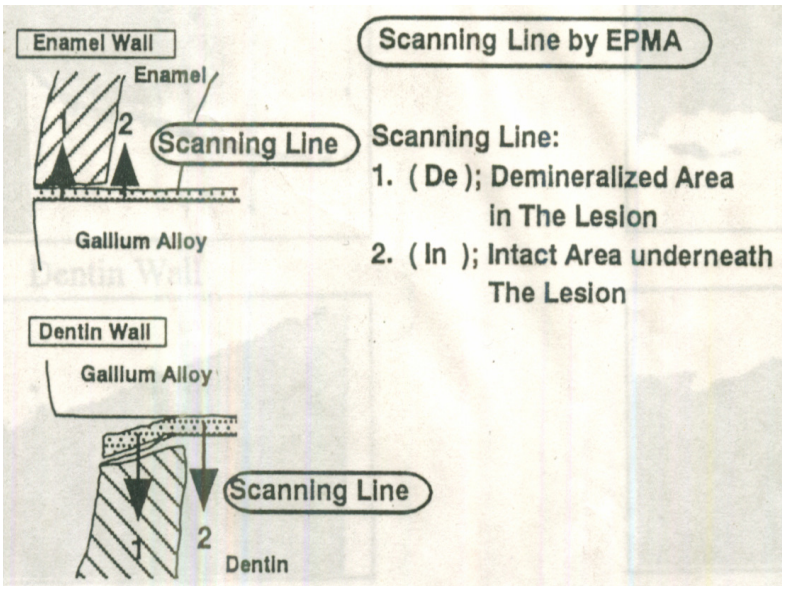

Figure 3. The sites of scanning lines by electron probe microanalysis.

as strongly inhibited, moderately inhibited or not inhibited at the enamel and dentin wall. The classified data in each group was analyzed with a simple Chi-square analysis at the $\mathrm{P}=0.05$ level.
Microanalysis for $\mathrm{Ca}, \mathrm{P}$ and $\mathrm{F}$ distribution in tooth substrates

One of the sections in each of experimental and control groups was polished, covered by a thin layer of carbon and examined by an electron probe apparatus (JXA-733, JEOL, Tokyo,Japan) operated with an accelerating voltage of $15 \mathrm{kVp}$ and specimen current of $2 \times 10^{4} \mathrm{~A}$. A line-scan analysis of $\mathrm{Ca}, \mathrm{P}$ and $\mathrm{F}$ was done on a line which ran across a cavity wall at the demineralized area in the lesion and the intact area underneath the lesion shown in Fig. 3.

\section{RESULTS}

Tables 2 and 3 indicate the number of samples classified the presence of caries inhibition zones at enamel walls and dentin walls. At both the enamel walls and dentin walls, $G$ was statistically different from ARG, ACG was different from ARG, and RG was different from ACG at $p<0.05$.

Caries inhibition zones presented in all samples at enamel walls adjacent to RG of group 2 and ARG of group 3, and at dentin walls next to RG of group 2 and ARG of all groups.

Fig. 4 shows the typical appearance of the lesions in each group by microradiography. In Fig. 4-B, wall lesions were detected at the enamel and dentin lesions adjacent to the cavity restored with $G$ of group 1. Only the outer lesions could be seen at the ACG control cavity, but caries inhibition zones were not be observed (Fig. 4-D). At the RG control cavity and the ARG experimental cavity, the lesions of the enamel and dentin wall definitely demonstrated the caries inhibition zones.

Fig. 5 shows the line analysis at the abovementioned sites with the same specimens. In all enamel and dentin lesions, the peaks of the $\mathrm{Ca}$ and $\mathrm{P}$ contents were revealed at cavity walls. Particularly, the counts of $\mathrm{Ca}$ and $\mathrm{P}$ in the inhibition zones were the same high level as that in intact areas at the RG control cavity and the ARG experimental cavity.

\section{DISCUSSION}

If restorations have poor marginal integrity, marginal gaps may extend deeper into the interface and develop secondary caries with 
Table 2. Number of samples classified the presence of caries inhibition zones at enamel wall.

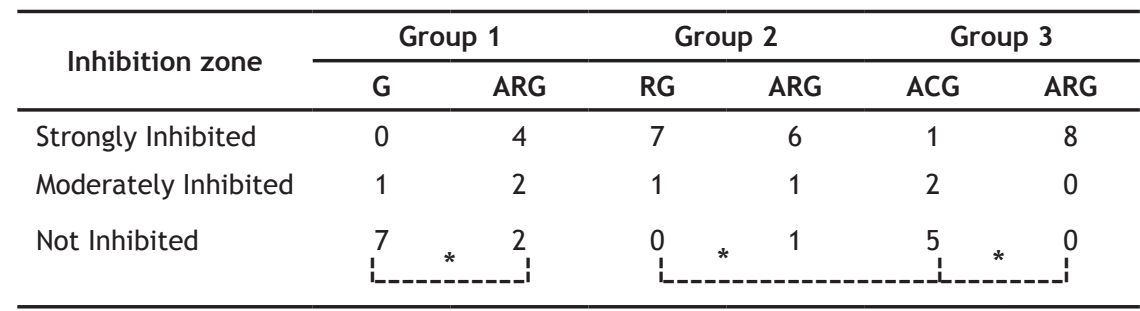

*: Significant difference by Chi-square analysis $(\mathrm{p}<0.05)$, (Number of samples in each group=8)

Table 3. Number of samples classified the presence of caries inhibition zones at dentin wall.

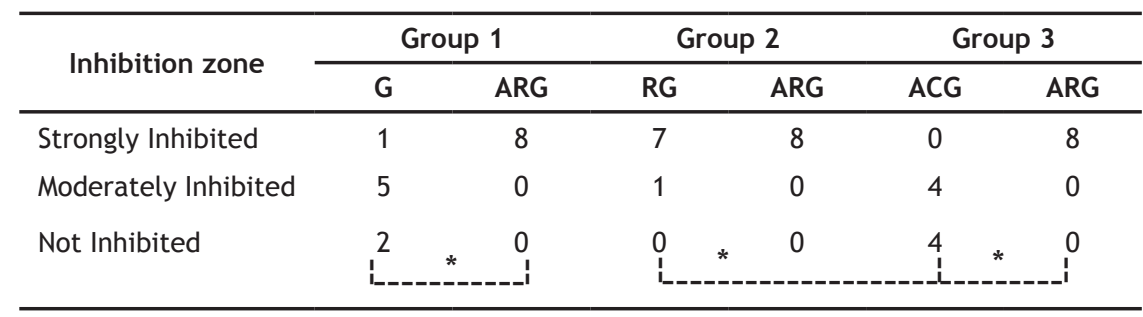
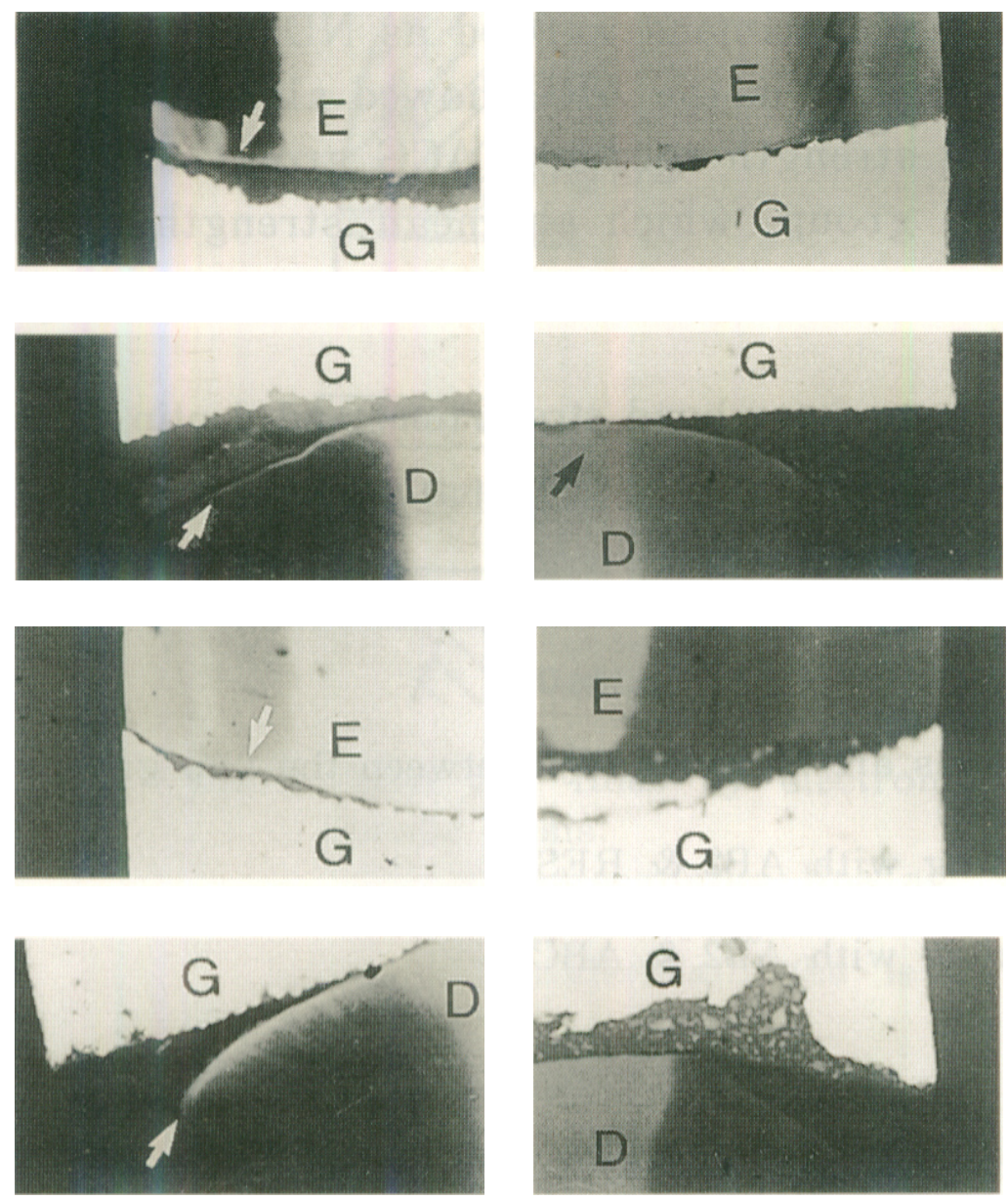

Figure 4. Microradiography of the typical lesions.

not only outer lesions but also wall lesions. The strategy for secondary caries inhibition around amalgam restorations is usually of the following methods: (1) Reinforcement of cavity walls by treatment with fluoride $;$ (2). Incorporation of fluoride in amalgam ${ }^{8}$; and (3). Bonding of amalgam to tooth structures treated by an adhesive agents. ${ }^{4}$ In this study, we studied application of fluoride 

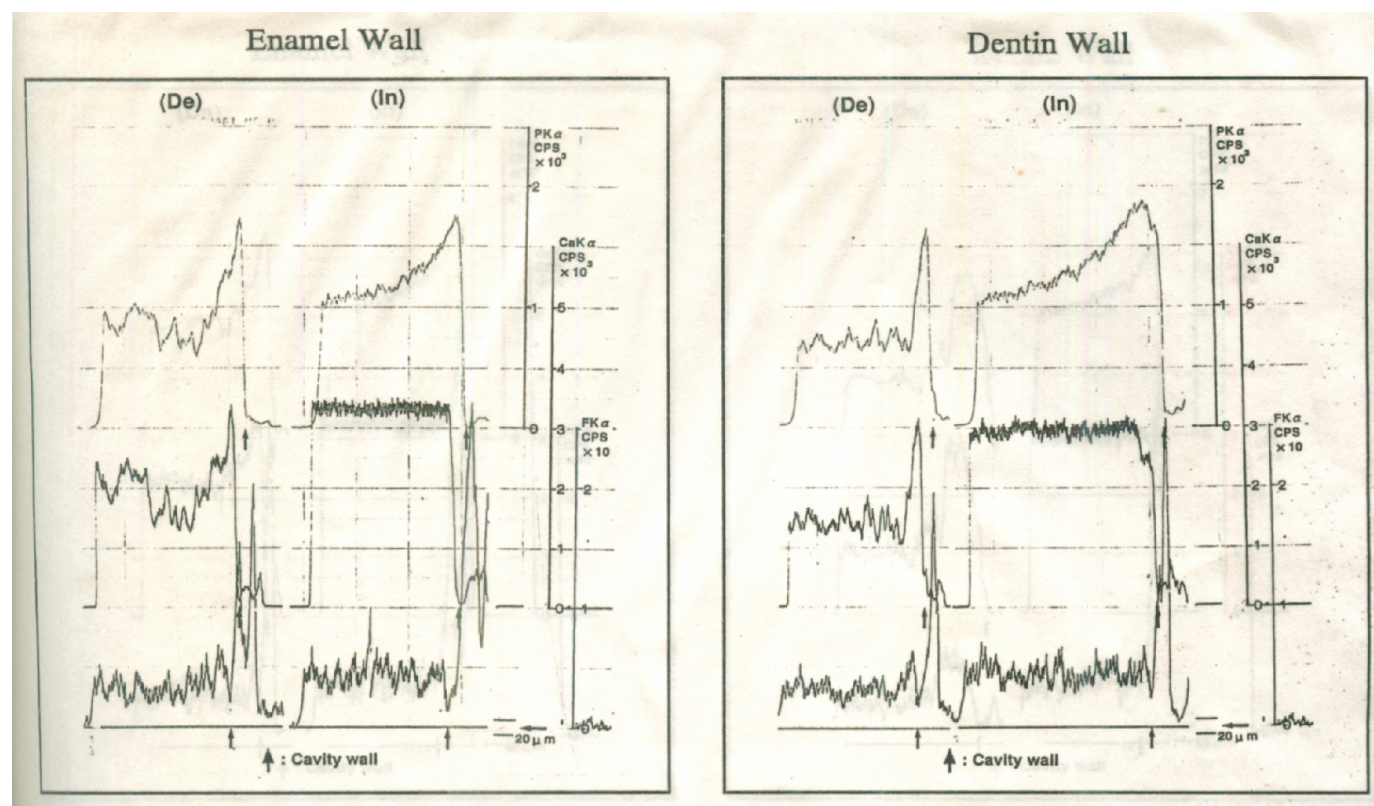

A:Experimental Cavity (ARG)
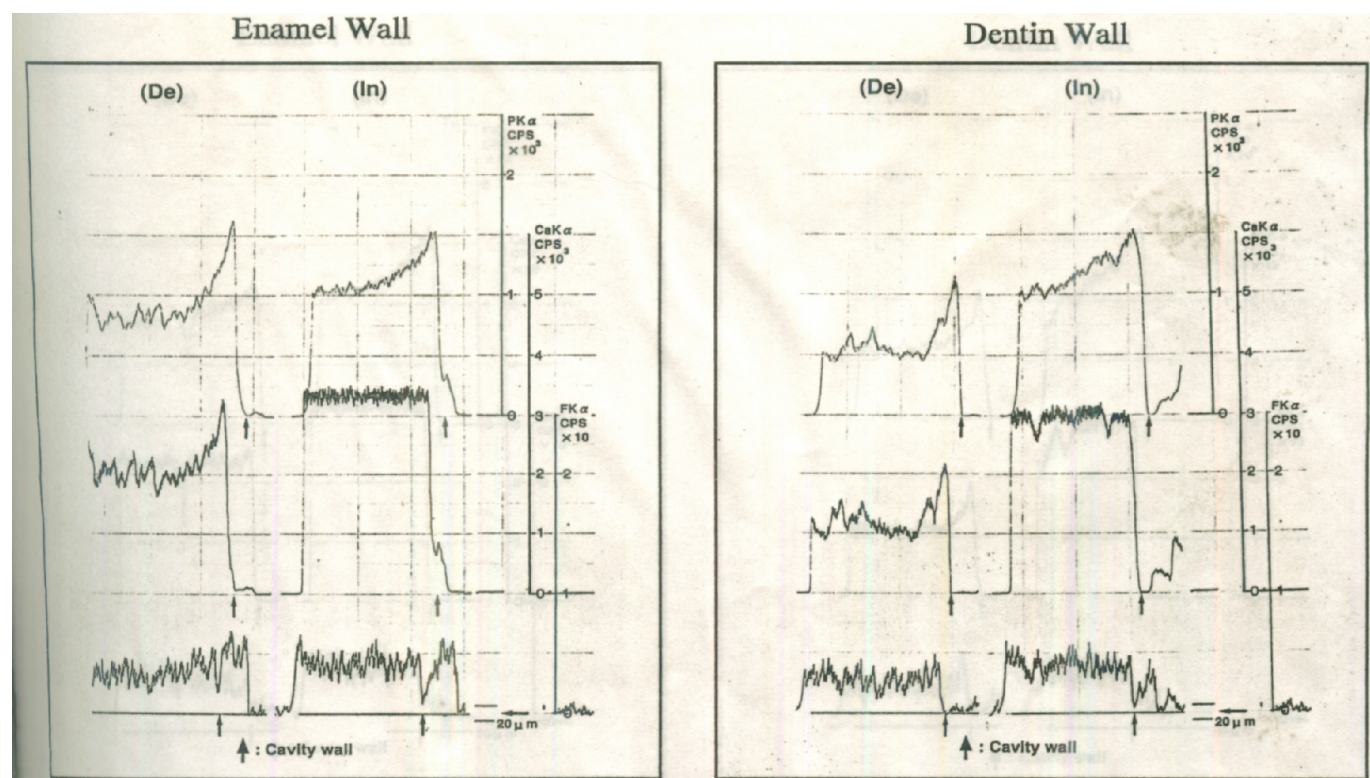

B:Control cavity (G)

Figure 5. The distributions of $\mathrm{Ca}, \mathrm{P}$ and $\mathrm{F}$ counts in the same samples as Figure 4.

releasing liners with bonding of amalgam to tooth structure.

In Fig. 4 Presinomer adhered to the dentin wall in the sample of ARG group, while all-bond C\&B of ACG group separated from the dentin wall and preferentially adhered to the gallium alloy. The application of Presinomer with All-bond 2 achieved high shear bond strength between amalgam and dentin $^{5}$, and produced more effective reduction of microleakage than adhesive resin cement. ${ }^{8}$ This results suggest that All-bond 2 and Presinomer are improved the marginal integrity in the gallium alloy restoration.

The caries inhibition zones in the RG and ARG groups were clearly demonstrated in Fig. 4-A and $\mathrm{C}$. In Fig. 5, the high $\mathrm{Ca}$ and $\mathrm{P}$ concentrations were noted, corresponding to the areas of the caries inhibition zones. The levels of $\mathrm{Ca}$ and $\mathrm{P}$ in the inhibition zones is similar to the intact areas. Nagamine et al. ${ }^{6}$ reported the high $\mathrm{Ca}$ and $\mathrm{F}$ concentrations is similar to the thickness of the caries inhibition zones in the demineralized dentin wall adjacent to restorative resin-modified glass ionomers. Presinomer contains sodium- 

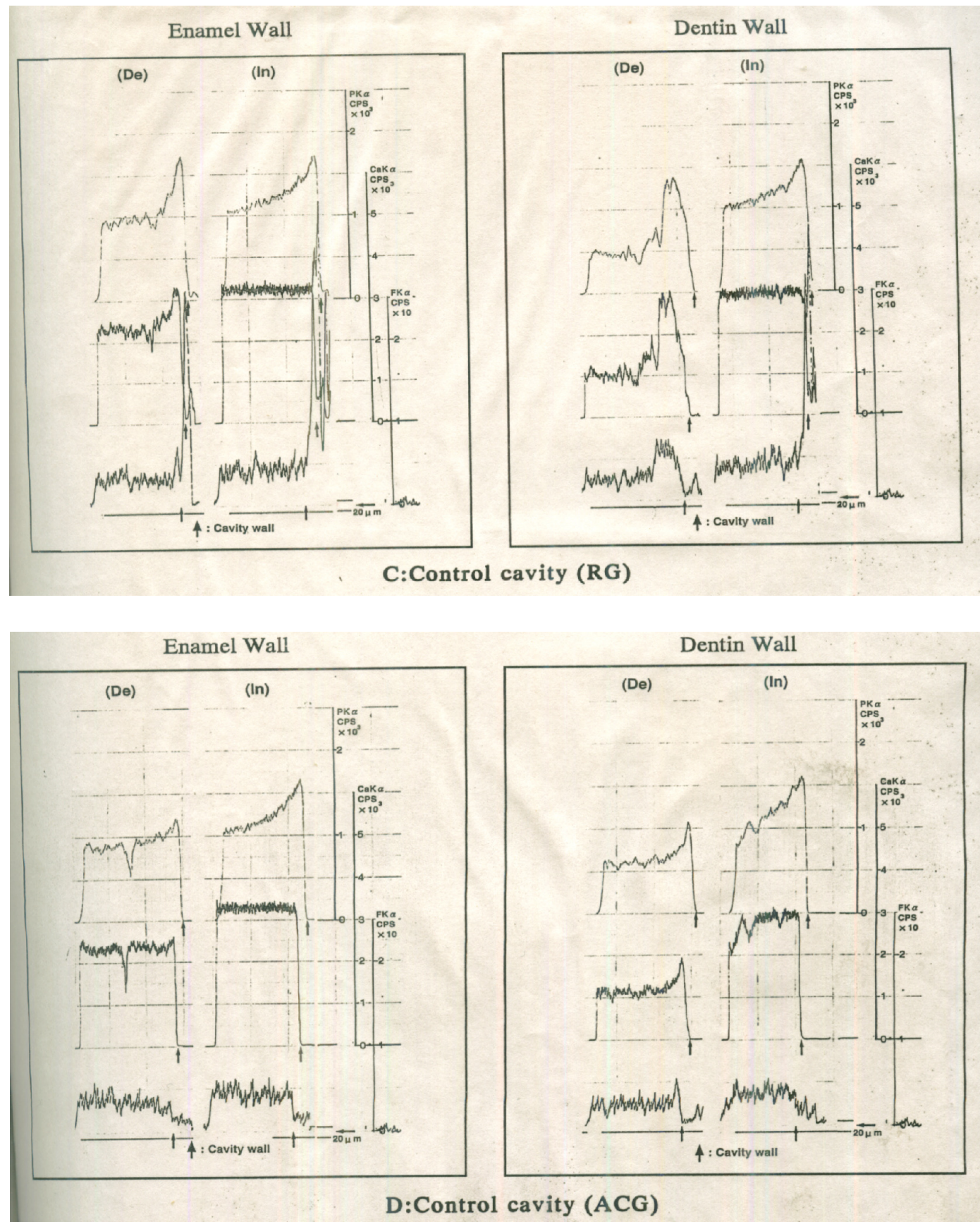

Figure 5. The distributions of $\mathrm{Ca}, \mathrm{P}$ and $\mathrm{F}$ counts in the same samples as Figure 4.

calcium-almino-fluorosilicate glass in the base, and gradually release the same volume of fluoride for long tenn as resin-modified glass ionomers. ${ }^{10}$ Therefore, fluoride released from Presinomer may be incorporated into tooth structure surrounding the restoration margins, resulting in the inhibition of dissolution from acid attack.

In addition, it was observed that the high $\mathrm{Ca}$ and $P$ counts in the inhibition zone at the RG dentin cavity wall extended into deeper areas than that at the ARG dentin cavity wall. Two percent NTGGMA (Ntolyglycine glycidyl methacrylate) and 16\%
BPDA (Biphenyl dimethacrylate) included in allbond 2 primer $A$ and $B$ may interfere the migration of fluoride ion. The inhibition zone should be evaluated by quantitative analysis.

The marginal seal at the gallium alloy-tooth interface depends upon good physical properties and dimensional change of $0.07 \% .{ }^{11}$ Potential benefits with All-bond 2 and Presinomer would be improved marginal seal, improved retention and reinforcement of tooth structure. However, the long term clinical effectiveness of the restoration in this system has not been reported and some 
potential problems would have to solved: it is difficult to prevent the discoloration of gallium alloy in clinical use and the bonding materials may be incorporated into the gallium alloy, weakening the marginal areas around the restorative materials.

\section{CONCLUSION}

The caries inhibition zones of enamel and dentin walls adjacent to gallium alloy restorations were clearly visible when Presinomer was used as a cavity liner with or without the All-bond 2 Bonding system. The amounts of $\mathrm{Ca}$ and $\mathrm{P}$ in the inhibition zones were comparable to the amounts in intact areas. The results indicated that the fluoride releasing resin-ionomer cement provided caries inhibition with or without etching and bonding, and that etching and bonding alone is not as effective as fluoride release.

\section{REFERENCES}

1. Hals E, Simonsen TL. Histopathology of experimental in vivo caries around silver amalgam fillings. Caries Res 1972;6:16-33.

2. Goldberg J, Amara J, Tanzer J, Thai F, Munster E, Birkhed D. Cross-sectional evalation of recurrent enamel caries, restoration of marginal integrity, and oral hyginee status. JADA 1981;102:635-41.

3. Kidd EM, O'hara J. The caries status of occlusal amalgams with marginal defects. J Dent Res
1990;68:1275-7.

4. Toni $Y$, Staninec $M$, Kawakami M, Imazato $S$, Toni M, Tsutitani Y. Inhibition in vitro of caries around amalgam restorations by bonding amalgam to tooth structure. Oper Dent 1989;14:142-8.

5. Kline J, Boyer D. Bond strength of amalgam to dentin with filled adhesives. J Dent Res 1996;75:175.

6. Nagamine $M$, Itota $T$, Toni $Y$, Inie $M$, Inoue K. Effect of light-cured glass ionomers on secondary caries in vitro. J Dent Res 1994;73:133.

7. Heintze V, Mornstad H. Artificial carieslike lesions around conventional, fluoride containing and dispersed amalgams. Caries Res 1980;14:414-21.

8. Tangsgoolwatana J, Cochran MA, Moore BK. Li Y. Microleakage evaluation of bonded amalgam restorations: Confocal microscopy versus radioisotope. J Dent Res 1996;75:172.

9. Alexander WE, McDonald RE, Stookey GK. Effect of stannous fluoride and recurrent caries-results after 24 months. J Dent Res 1973;52:1147.

10. Burgess JO. Dental materials for the restoration of root surface caries. Am J Dent 1995;8:34251.

11. Okamoto Y, Naruse S, Yamamoto H. Influence of $\mathrm{Pd}$ addition on the physical properties of gallium filling materials. J Fukuoka Dent Coll 1992;19:347-52. 